\title{
TRATAMENTO CIRÚRGICO DAS HÉRNIAS INGUINAIS SOB ANESTESIA LOCAL EM AMBULATÓRIO
}

\section{INGUINAL HERNIORRHAPHY WITH LOCALANESTHESIAAS AN OUTPATIENT PROCEDURE}

\author{
Rafael Melillo Laurino Neto, TCBC-SP ${ }^{1}$ \\ Andréa Alessandra Callas Marino Buchmann ${ }^{2}$ \\ Luis Renato Rotta Messias ${ }^{2}$
}

\begin{abstract}
RESUMO: Objetivos: Apresentar o programa e avaliar a tolerância dos pacientes à correção das hérnias inguinais em ambulatório sob anestesia local. Método: Foram analisados 61 pacientes submetidos a tratamento cirúrgico de hérnia inguinal unilateral não complicada, sob anestesia local em ambulatório no Serviço de Cirurgia Geral do Conjunto Hospitalar do Mandaqui, entre fevereiro de 2000 e agosto de 2002. Respeitandose rígidos critérios na seleção dos pacientes, e com os mesmos sob sedação e monitorização contínua, realizou-se bloqueio de campo conforme padronização do serviço. A técnica de reforço da parede abdominal foi definida levando-se em consideração o tipo de hérnia, idade do paciente e sua atividade profissional. Todos os pacientes receberam alta hospitalar em até quatro horas após a cirurgia respeitando-se as condições estabelecidas pela resolução $C F M n^{\circ} 1.409 / 94$, com orientações precisas quanto a possíveis complicações imediatas e retorno para atendimento em Pronto-Socorro caso necessário. Resultados: O tempo cirúrgico médio foi de $1 \mathrm{~h} 30 \mathrm{~min}$. Quanto ao tipo de hérnia, segundo a classificação de Nyhus, prevaleceu o tipo III B (36\%), seguido dos tipos III A $(34,5 \%)$ e II $(26,2 \%)$. A técnica de reforço mais utilizada foi a de Lichtenstein $(80,3 \%)$. Quanto à avaliação da dor intra-operatória, $73,8 \%$ dos pacientes deram notas igual ou inferior a 3 numa escala de 0 a 10 e $95 \%$ afirmaram que se submeteriam novamente a tal procedimento sob anestesia local. Conclusões: $\mathrm{O}$ tratamento cirúrgico das hérnias inguinais sob anestesia local em ambulatório, é procedimento seguro e bem aceito pela maioria dos pacientes quando realizado de forma padronizada.
\end{abstract}

Descritores: Hérnia inguinal; Procedimentos cirúrgicos ambulatorial; Anestesia local.

\section{INTRODUÇÃO}

O tratamento das hérnias inguinais deve ser feito com base no conhecimento das estruturas anatômicas e de suas relações nesta região tendo como principais objetivos: mínima recidiva, poucas complicações operatórias e o retorno mais rápido possível do paciente às suas atividades habituais. No início do século, o tempo médio de internação para uma cirurgia de hérnia inguinal era de vinte e um dias. Com o aprimoramento das técnicas cirúrgicas e anestésicas e com a tendência crescente à não hospitalização, o período de internação se reduziu, havendo atualmente a possibilidade de realizá-la em regime ambulatorial, frequentemente com a utilização de anestesia local. A cirurgia ambulatorial tem diminuído o tempo de recuperação e o custo, tanto econômico quanto social dos procedimentos, evitando as complicações inerentes à permanência hospitalar prolongada, além de suprir a necessidade crescente de leitos hospitalares. ${ }^{1-12}$ A utilização de anestesia local no reparo destas hérnias, antecede ao conceito de cirurgia ambulatorial, pois vem sendo empregada com esta finalidade há mais de cinquenta anos. ${ }^{13-15}$ Seguindo esta tendência e uti-

1. Cirurgião do Conjunto Hospitalar do Mandaqui TCBC-SP.

2. Ex-Residente de Cirurgia Geral do Conjunto Hospitalar do Mandaqui.

Recebido em 07/05/2003

Aceito para publicação em 16/03/2004

Trabalho realizado no Serviço de Cirurgia Geral do Conjunto Hospitalar do Mandaquí - São Paulo (SP). 
lizando a estrutura de uma Unidade de Cirurgia Ambulatorial Autônoma ${ }^{2-16}$ passou-se a realizar a correção cirúrgica da hérnia inguinal sob anestesia local com alta precoce a partir do ano de 2000 no Conjunto Hospitalar do Mandaquí.

O objetivo deste trabalho é apresentar os resultados iniciais desta padronização de tratamento, avaliando a aceitação pelos pacientes do método anestésico e resultados cirúrgicos.

\section{MÉTODO}

Foram estudados 61 pacientes submetidos a correções cirúrgicas de hérnia inguinal sob anestesia local no Centro Cirúrgico do Ambulatório do Conjunto Hospitalar do Mandaquí no período de fevereiro de 2000 a agosto de 2002 . A seleção dos pacientes para o procedimento obedeceu rigorosamente aos seguintes critérios de exclusão $5,6,17,18$ : idade menor que 13 anos, ASA > 2, tossidores crônicos, portadores de distúrbios psiquiátricos graves, convulsivos crônico, obesos(índice da massa corpórea $>35 \mathrm{~kg} / \mathrm{m} 2$ ), usuários de anticoagulantes, antidepressivos tricíclicos, inibidores da monoamino oxidase ou drogas ilícitas, grandes defeitos anatômicos, hérnias encarceradas , estranguladas ou por deslizamento, hérnias femorais ou recidivadas.

Durante a primeira consulta, os pacientes selecionados eram apresentados ao protocolo para formalizarem consentimento ao procedimento e realizarem os exames pré-operatórios. Após o preparo pré operatório, os pacientes eram orientados a comparecer ao Centro Cirúrgico Ambulatorial no dia da operação em jejum de oito horas, com duas horas de antecedência à cirurgia, com acompanhante maior, lúcido e previamente identificado, quando tinha seus parâmetros vitais avaliados, observando-se a inexistência de sintomas e sinais de doença aguda, principalmente respiratória e de lesões cutâneas na região a ser operada. Realizava-se então tricotomia inguinal pouco antes do ato operatório ${ }^{2,3,8}$.

$\mathrm{Na}$ sala cirúrgica, os pacientes eram devidamente monitorizados, instalando-se máscara de $\mathrm{O}_{2} \mathrm{e}$ acesso venoso periférico por onde realizava-se infusão de medicação pré-anestésica (Midazolan-0,1mg/ $\mathrm{kg}$ endovenoso) ${ }^{4,17}$. Mantinha-se obrigatoriamente na sala de operação todo o material necessário para tratamento das possíveis complicações clínicas e anestésicas. Como anestésico foi padronizada uma solução alcalinizada de lidocaína a $0,5 \%$ e bupivacaína a $0,125 \%{ }^{4,6,9}$. Optou-se por realizar bloqueio regional seguido de infiltração local nos seguintes pontos ${ }^{4,6,13}$ :

- ponto localizado $2 \mathrm{~cm}$ superior e medialmente à espinha ilíaca ântero-superior com infiltração em leque de plano subaponeurótico.

- projeção do tubérculo púbico, penetrandose a agulha perpendicularmente até tocar o periósteo do ramo superior do púbis.

- linha imaginária, paralela e lateral ao ligamento inguinal unindo o primeiro ao segundo pontos de punção.

- pele e tecido subcutâneo no local de incisão.

- após incisão de pele tecido subcutâneo, complementava-se a anestesia com infiltração subaponeurótica do músculo oblíquo externo, a partir do anel inguinal externo sob visão direta.

- dissecado o saco herniário, infiltrava-se o colo do mesmo na altura do ânulo inguinal profundo antes de completar a dissecção e efetuar a ligadura deste.

A técnica para reforço da parede abdominal era definida no intra operatório, levando-se em consideração o tipo de hérnia, idade do paciente e sua atividade profissional. Utilizou-se neste trabalho a classificação de hérnias inguinais de Nyhus, que se baseia em critérios anatômicos (tamanho do anel interno e integridade da parede posterior do canal), classificando as hérnias em quatro tipos. ${ }^{19}$

Realizou-se neste grupo a correção das hérnias pelas técnicas de Bassini modificado, Lichtenstein e Marcy. Ao término da cirurgia os pacientes permaneciam na sala de recuperação pós-anestésica por aproximadamente quatro horas aonde recebiam dieta leve quando bem acordados e realizavam-se controles periódicos de seus parâmetros hemodinâmicos e clínicos.

Os pacientes recebiam alta hospitalar quando preenchiam os critérios estabelecidos pela Resolução CFM n ${ }^{\circ}$ 1409/94, ou seja:

- orientação no tempo e no espaço

- estabilidade dos sinais vitais por pelo menos $60 \mathrm{~min}$

- ausência de náuseas ou vômitos

- ausência dificuldade respiratória

- capacidade de ingerir líquidos

- ausência de dor de grande intensidade

- ausência de sinais de retenção urinárias

- capacidade de se locomover como antes da cirurgia

Recebiam então instruções verbais e por escrito a respeito de cuidados com a ferida operatória, 
realização de curativo, uso de medicações, evolução normal do pós-operatório e principais complicações locais e sistêmicas, sendo orientados a procurar o Pronto-Socorro do Hospital caso fosse necessário. O primeiro retorno ambulatorial era marcado para o $7^{\circ}$ dia de pós-operatório, seguido de outros no $14^{\circ}$ e $30^{\circ}$ dias, sendo então acompanhados até um ano de pósoperatório com intervalos de dois meses. O início da coleta de dados se dava antes da alta do paciente, quando este respondia a um questionário para avaliação da dor intraoperatória a qual era quantificada segundo escala analógica com uma nota de 0 a 10, sendo zero ausência de dor e 10 a dor mais intensa que o indivíduo já sentira na vida. Perguntava-se também neste momento, se caso o paciente tivesse uma hérnia contralateral, se este se submeteria ao mesmo procedimento novamente 5 .

Registrou-se no seguimento ambulatorial o período que o indivíduo permanecera com dor e sua intensidade e o tempo de retorno às atividades, bem como complicações imediatas e tardias da cirurgia.

\section{RESULTADOS}

No grupo estudado, 58 indivíduos (95\%) eram do sexo masculino e três (5\%) do feminino, com média de idade de 35,5 anos variando de 13 a 62 anos. O tempo cirúrgico médio foi de $1 \mathrm{~h}$ e 30 min com tempo mínimo de 50 min e máximo de 2 hs e $10 \mathrm{~min}$.

Os tipos de hérnia encontradas, segundo a classificação de Nyhus foram duas $(3,3 \%)$ do tipo I, $16(26,2 \%)$ do tipo II, $21(34,5 \%)$ do tipo III A e 22 (36\%) do tipo III B. A técnica de reforço mais utilizada foi a de Lichtenstein em 49 pacientes $(80,3 \%)$, seguida da de Bassini modificado em 10 pacientes $(16,4 \%)$ e Marcy em dois pacientes (3,3\%).

Em relação à intensidade da dor intra operatória, 17 pacientes $(27,9 \%)$ deram nota 0,10 pacientes $(16,4 \%)$ nota 1 , oito pacientes $(13,1 \%)$ nota 2 , 10 pacientes $(16,4 \%)$ nota 3 , quatro pacientes $(6,6 \%)$ nota 4 , seis pacientes $(9,8 \%)$ nota 5 , cinco $(8,2 \%)$ nota 6 e um paciente $(1,6 \%)$ nota 8 . Observa-se com estes números que $73,8 \%$ dos indivíduos analisados quantificaram sua dor com uma nota menor ou igual a 3 o que caracteriza nesta escala dor inexistente ou leve. Dos pacientes analisados, 58 (95\%) relataram que se submeteriam a tal procedimento novamente sob anestesia local se tivessem uma nova hérnia contralateral.
Avaliando-se o período pós-operatório precoce, cinco pacientes $(8,2 \%)$ referiram dor de média intensidade que cessou com analgésicos, dois pacientes $(3,3 \%)$ apresentaram formação de hematoma nos primeiros sete dias os quais foram acompanhados clinicamente sem necessidade de drenagem e um paciente $(1,6 \%)$ apresentou infecção de ferida operatória a qual foi tratada com antibióticos sistêmicos e curativos.

Na reavaliação do décimo quarto dia, apenas dois pacientes $(3,3 \%)$ mantiveram a sintomatologia dolorosa, a qual perdurou por vários meses caracterizando neuralgias persistentes. Em geral os pacientes estiveram aptos a retornar ao trabalho com restrições após 14 dias e sem as mesmas após quatro semanas da operação ${ }^{2,20}$.

Observou-se também a presença de duas recidivas $(3,3 \%)$ no primeiro ano de seguimento ambulatorial, os quais foram reoperados sob raquianestesia.

\section{DISCUSSÃO}

A utilização de anestesia local por bloqueio de campo, tem como premissas, a necessidade do conhecimento da anatomia da inervação da parede abdominal, e da ação farmacológica dos anestésicos locais, em especial do tempo de latência de cada droga e seus efeitos colaterais. É desejável que o anestésico para ser utilizado por infiltração ou bloqueio regional proporcione rápida instalação de anestesia, duração de efeito suficiente que permita a realização da operação, garanta período de analgesia no pósoperatório imediato, e não produza lesões teciduais.

Deve-se chamar a atenção para algumas complicações da anestesia local como reação vagal, tóxica, reação ao vasoconstritor e reações alérgicas, e para o fato de que determinadas drogas com a cimetidina e quinidina, e estados patológicos como Infarto Agudo Miocárdio e Insuficiência Pulmonar Crônica, aumentarem a toxicidade da lidocaína, interferindo em seu metabolismo. Nesta casuística, não se observou efeitos indesejáveis ou de difícil controle pelo uso dos anestésicos locais. Atribuiu-se tal fato ao respeito aos limites da dose de segurança das drogas, o que foi facilitado pela utilização dos anestésicos nas diluições de $0,5 \%$ para lidocaína e $0,125 \%$ para bupivacaína. O uso da solução de anestésicos, permitiu a associação das características benéficas de ambas as drogas, ou seja, o início mais rápido de ação da lidocaína e duração prolongada da bupivacaína. 
Pode-se salientar ainda o fato da anestesia local envolver área limitada, que não interfere com o funcionamento de outros órgãos, permitindo relaxamento muscular localizado, não alterando a ventilação pulmonar e possibilitando a deambulação precoce. A anestesia local possibilitou no intra-operatório, testar a resistência do reforço, através da Manobra de Valssalva, bem como avaliar as condições do assoalho do canal inguinal.

O uso de medicação pré-anestésica Midazolan se mostrou benéfica porque além de produzir a sedação desejada, desencadeou também amnésia retrógrada com pequeno risco de agitação psicomotora. No pósoperatório destes pacientes, não se observou náuseas, vômitos, cefaléia e retenção urinária, proporcionando uma recuperação pós-anestésica mais curta, com redução do tempo de internação.Evitou-se operar sob anestesia local os grandes defeitos herniários e as hérnias de escorregamento, as quais possivelmente exigiriam dissecções extensas, pelas grandes áreas envolvidas com intensa manipulação das estruturas e pela maior quantidade de anestésico que se faria necessária no procedimento. Não foi recomendada também sua utilização em hérnias encarceradas, pela tração de vísceras e eventual necessidade de secção intestinal assim como também pelo tempo mais prolongado da operação. Pacientes obesos foram excluídos por apresentarem maiores dificuldades técnicas para o bloqueio loco-regional com difícil localização de pontos anatômicos de reparo para infiltração do anestésico assim como por necessitarem de doses maiores deste para atingir o efeito desejado.

Quanto ao reforço de parede abdominal, prevaleceu o uso de técnica sem tensão com uso de tela de polipropileno na maioria dos casos, pelas características da população estudada (trabalhadores braçais jovens), aos quais tal técnica ofereceu possibilidade de retorno mais precoce às atividades físicas intensas com menor índice de recidiva, não o fazendo apenas nos casos em que anatomia permitia a confecção de reforço tecidual com total segurança. $\mathrm{O}$ prazo mediano de retorno ao trabalho vem diminuindo de 50 a 60 dias na década de 70 para menos de 20 dias atualmente. Considera-se hoje, que a dor deva ser o guia para orientar o paciente a decidir se uma atividade física pode ser reiniciada ou não. Nos estudos mais recentes, observa-se que, não importando qual seja o método de correção, recomenda-se ao paciente não limitar sua atividade e fazer tudo o que se sentir apto e sem desconforto físico evitando apenas o que lhe provocar dor.$^{20,21}$

Não obstante os cuidados técnicos do procedimento, acreditamos ter sido a criteriosa seleção dos pacientes, fator decisivo para os resultados obtidos e satisfação daqueles que a ele se submeteram. Tornase evidente também, que com a implantação de unidades de cirurgia ambulatorial, a comunidade passa a dispor de mais leitos para internação de casos mais complexos, maior cobertura assistencial e aumento da relação custo eficiência das instituições.

\begin{abstract}
Background: To present the program and to evaluate the patient's tolerance to herniorrhaphy in an outclinic under local anesthesia. Methods: Between February 2000 and August 2002, 61 patients with non complicated unilateral inguinal hernia were treated under local anesthesia in an out-clinic at the General Surgery Service of the Mandaqui Hospital. Strict criteria to select patients were observed. Patients were sedated and under continuous monitoring local anesthesia was performed according to the service standardization. The technique to reinforce the abdominal wall was defined considering the type of hernia, age of patient and professional activity. All patients were discharged from hospital in up to 4 hours after the surgery, considering the conditions established by the CFM resolution NR 1.409/94 and observing the precise orientations in regard to possible immediate complications and return to the hospital, if needed. Results: The mean surgical time was 90 minutes. Regarding the type of hernia, according to the Nyhus classification, type III B (36\%) prevailed, followed by the types III A (34.5\%) and II (26.2\%). The most used technique was Liechtenstein's (80.3\%). Regarding intraoperative pain, in a scale from 0 to 10, 73.8\% of the patients gave marks equal or bellow 3 and $95 \%$ stated that they could be submitted to another herniorrhaphy under local anesthesia. Conclusions: the surgical treatment of inguinal hernia under local anesthesia in an out-clinic is a safe procedure and it is well accepted by most of the patients when performed in a standardized way.
\end{abstract}

Key Words: Hernia, inguinal; Ambulatory surgical procedures; Anesthesia, local. 


\section{REFERÊNCIAS}

1. Glassow F - Short-stay surgery (Shouldice technique) for repair of inguinal hernia. Ann R Coll Surg Engl, 1976, 58(2):133-139.

2. Oliva Filho AL - Anestesia para pacientes de curta permanência hospitalar. Anestesia ambulatorial. Rev Bras Anestesiol, 1983, 33(1):51-62.

3. Balbinot AL, Espíndola MB, Junqueira Jr G, et al. Hernioplastia inguinal e femoral com anestesia local em nível ambulatorial. Rev Col Bras Cir, 1997, 24(6):427430.

4. Rodrigues Jr AJ, Mittelstaedt WEM, Bevilacqua RG, et al. - Anestesia local por bloqueio de campo no tratamento ambulatorial das hérnias inguinais. Rev Col Bras Cir, 1992, 19(5):195-198.

5. Flanagan L, Bascom JU - Herniorrhaphies performed upon outpatients under local anesthesia. Surg Gynecol Obstet, 1981, 153(4):557-560.

6. Henriques AC, Pezzolo S, Silva GG, et al. - Herniorrafia inguinal sob anestesia local. Rev Col Bras Cir, 1997, 24(6):405-408.

7. Abdu RA - Ambulatory herniorrhaphy under local anesthesia in a community hospital. Am J Surg, 1983, 145(3):353-356.

8. Monteiro MC, Ferreira RA - "Tratamento ambulatorial das hérnias da parede abdominal". In Souza JAG, Silva AO (eds) - Cirurgia ambulatorial. Rio de Janeiro. Atheneu, 1999, pp. 83-92.

9. Wantz GE - Ambulatory hernia surgery. Br J Surg, 1989, 76(12):1228-1229.

10. Velez-Gil A, Wilson D, Pelaez RN - A simplified system for surgical operations: the economics of treating hernia. Surgery, 1975, 77(3):391-394.

11. Coe RC - Inguinal herniorrhaphy under local anesthesia. Arch Surg, 1978, 113(7):905.

12. Oliveira MR, Speranzini MB, Junqueira Jr A - A atualidade da cirurgia ambulatorial. Rev Bras Educ Méd, 1985, 9(1):52-54.
13. Tani F, Coratti A, De Martino A, et al. - L'anestesia locoregionale nella chirurgia dell'ernia inguinale. Minerva Anestesiol, 2000, 66(4):201-206.

14. Oliva Filho AL - Conceito de anestesia ambulatorial. Rev Bras Anestesiol, 1983, 33(2):107-116.

15. Glassow F - Inguinal hernia repair using local anaesthesia. Ann R Coll Surg Engl, 1984, 66(6):382387.

16. Speranzini MB, Deutsch CR, Lima Jr. SE - "Organização e implantação de um serviço de cirurgia ambulatorial: tipos de unidades ambulatoriais". In Souza JAG, Silva AO (eds) - Cirurgia Ambulatorial. Rio de Janeiro. Atheneu, 1999, pp. 11-17.

17. Pritchard TJ, Bloom AD, Zollinger RM - Pitfalls in ambulatory treatment of inguinal hernias in adults. Surg Clin North Am, 1991, 71(6):1353-1362.

18. Minossi JG, Pedro FAJ, Vendites S - Herniorrafia inguinal com anestesia local. Arq Gastroenterol, 1992, 29(1):18-22.

19. Rutkow IM, Robbins AW - Demographic, classificatory, and socioeconomic aspects of hernia repair in the United States. Surg Clin North Am, 1993, 73(3):413-426.

20. Callessen T, Klarskov B, Bech K, et al. - Short convalescence after inguinal herniorrhaphy with standardised recommendations: duration and reasons for delayed return to work. Eur J Surg, 1999, 165(3):236241.

21. Baskerville PA, Jarrett PE - Day case inguinal hernia repair under local anaesthetic. Ann R Coll Surg Engl, 1983, 65(4):224-225.

Endereço para correspondência:

Rafael M. Laurino Neto

Rua Cotoxó 611 cj. 98 Pompéia

São Paulo ( SP) 05021-001

E-mail:rmelillo@uol.com.br 